\title{
Theory of magnetization dynamics in a dual-free-layer spin-torque nano-oscillator with isotropic free layers
}

\author{
O.V.Prokopenko, V.S.Tiberkevich ${ }^{*}$, A.N. Slavin* \\ Faculty of Radiophysics, T.Shevchenko National University of Kyiv, \\ 64/13 Volodymyrska Str., 01601 Kyiv, Ukraine \\ "Department of Physics, Oakland University, 2200 North Squirrel Rd., \\ 48309 Rochester, MI, USA
}

Received October 10, 2013

\begin{abstract}
The analytical theory of magnetization dynamics in a dual-free-layer spin-torque nanooscillator (DFL STNO) operating in the absence of a bias magnetic field is developed. The theory is based on a system of simplified dynamic equations written in collective variables and yields an analytic expression for the upper dc current density threshold of stable magnetization dynamics (generation) in the DFL STNO. This threshold is caused by a dipolar coupling between the free layers and can be controlled by choosing an appropriate geometry of the DFL STNO structure.
\end{abstract}

Разработана аналитическая теория динамики намагниченности, происходящей в отсутствие внешнего постоянного подмагничивающего поля, в спинтронном наноосцилляторе (СНО) с двумя свободными магнитными слоями. Теория основана на системе двух упрощенных уравнений, записанных для коллективных переменных и позволяет получить аналитическое выражение для верхнего порога стабильной динамики намагниченности (генерации) в СНО. Показано, что этот порог генерации определяется наличием дипольной связи между свободными слоями и может быть контролируемым за счет выбора соответствующей геометрии СНО структуры.

Теорія динаміки намагніченості у спінтронному наноосциляторі 3 двома ізотропними вільними магнітними шарами. О.В.Прокопенко, В.С.Тиберкевич, А.М.Славін.

Розроблено аналітичну теорію динаміки намагніченості, що відбувається за відсутності зовнішнього постійного поля підмагнічування, у спінтронному наноосциляторі (CHO) з двома вільними магнітними шарами. Теорія базується на системі двох спрощених рівнянь, записаних для колективних змінних, і дозволяє отримати аналітичний вираз для верхнього порогу стабільної динаміки намагніченості (генерації) у СНО. Показано, що цей поріг генерації визначається наявністю дипольного зв'язку між вільними шарами і може бути контрольованим за рахунок вибору відповідної геометрії СНО структури.

\section{Introduction}

The spin-torque nano-oscillators (STNOs), based on the spin-transfer torque (STT) [1,2] effect, are now considered as promising base elements for various nanoscale microwave devices [3], in particular, microwave signal sources [4-6] and micro- wave detectors [7-12]. However, the output power, operation frequency and phase noise of these devices must be improved in order to use them in practical applications [13, 14].

A new type of an STNO that comprises two outermost out-of-plane (OOP) pinned magnetic layers (PLs) serving as perpendicular polarizers and two inner free mag- 
netic layers (FLs) with in - plane easyplane anisotropy - a dual-free-layer (DFL) STNO (Fig. 1(a)) - has been proposed in [15] and then studied numerically in [16-18].

In the presence of a direct bias current of the density $J_{d c}$ applied across the entire stack, as it is shown in Fig. 1(b), the perpendicular polarizers provide a STT, $\mathbf{T}_{S, j}$, to the adjacent FLs and push their magnetic moments out of the $(x, y)$ plane towards the opposite ends of the structure: $m_{z, b}<0$, $m_{z, t}>0$ for $J_{d c}>0$ (current passing the structure from bottom to top, while the electrons are moving in opposite direction). Here $m_{z, i}<0$ is the $z$-component of the unit vector $\mathbf{m}_{i}$ directed along the magnetization of the $i$-th FL, $i=t$ for the top layer and $i=b$ for the bottom layer, respectively. The Gilbert damping torque, $\mathbf{T}_{D, j}$, opposes this action and pushes the magnetizations back towards the plane. Easy-plane demagnetizing fields, that arise in both layers in proportion to their OOP magnetization components, induce the FLs' magnetic moments to precess in opposite directions around the normal to the sample plane (torque $T_{p}$ ) [19]. In such a geometry it is possible to achieve the opposite directions of the current-driven magnetization precession in the FLs, and, therefore, to double of the generated microwave frequency [15-18].

In this work we present an analytical theory of the operation of a symmetric DFL STNO with identical isotropic FLs. We obtain the simplified equations describing the magnetization dynamics in dipolarly coupled FLs of the DFL STNO and analytically calculate the upper threshold of the stable dynamics in the hysteresis regime of the DFL STNO operation recently observed in $[17,20]$. These results might be important for the development of high-power high-frequency STNOs operating in the absence of an external magnetic field.

\section{Theory}

We consider a simple model of a symmetric DFL STNO, formed by a circular nanopillar consisting of two thin identical isotropic FLs of the thickness $L$, two identical PLs and three identical metallic GMR spacers of the thickness $d$ (see Fig. 1(a)). The radius $R$ of the nano-pillar is assumed to be sufficiently small, so that the magnetizations of all magnetic layers are spatiallyuniform and can be treated in the macrospin approximation. The magnetizations of the PLs of the STNO are assumed to be completely fixed and lie perpendicular to

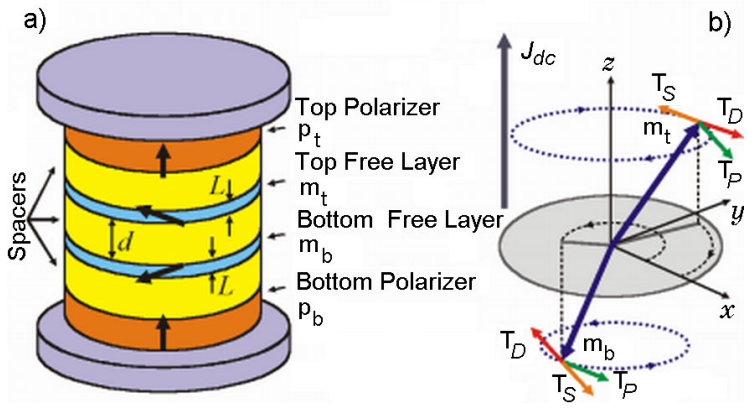

Fig. 1. (a) Layout of the symmetric dual-free layer (DFL) STNO consisting of four magnetic layers: two inner free magnetic layers (FLs) of the thickness $L$ and two outer pinned layers with perpendicular polarization separated by the GMR spacer of the thickness $d$. (b) The torques acting on the magnetization vectors of the FLs in the DFL STNO driven by a transverse bias dc current of the density $J_{d c}$.

the layers' plane - their direction $\mathbf{p}=\mathbf{z}$ is determined by the perpendicular magnetic anisotropy of the polarizers' material. The FLs of the DFL STNO are dipolarly coupled. The STNO operates in the absence of an external bias $d c$ magnetic field, so that the effective magnetic field acting on the FLs has contribution from the self- and cross-demagnetization fields only. For simplicity an easy-axis anisotropy field, which is included in the numerical experiments $[16,17,20]$, is excluded from the theoretical analysis. As it was shown in [17, 20], this anisotropy mainly influences only the value of the upper and lower $d c$ current density thresholds.

Numerical simulations reported in [16, 20] reveal that the STT related coupling between the FLs has negligible effect on the dynamics, and, hence, the STT interaction between the FLs was not included in the theory. We also found that the dipolar field originating from the pair of polarizers, also, has a negligible effect on the dynamics, and, therefore, the dipolar coupling between the FL and other PLs is omitted from our theoretical analysis.

The dynamics of the unit magnetization vectors $\mathbf{m}_{i}=\mathbf{M}_{i} / M_{S}$ (here $M_{S}$ is the saturation magnetization) in the $i$-th FL under the action of a bias $d c$ current density $J_{d c, i}$ is governed by the Landau-Lifshits-Gilbert equation [21] with Berger-Slonczewski STT term [1, 2] (LLGS equation): 


$$
\begin{gathered}
\frac{d \boldsymbol{m}_{\boldsymbol{i}}}{d t}=\gamma\left[\boldsymbol{B}_{\text {eff }, i} \times \boldsymbol{m}_{\boldsymbol{i}}\right]+ \\
+\alpha\left[\boldsymbol{m}_{\boldsymbol{i}} \times \frac{d \boldsymbol{m}_{\boldsymbol{i}}}{d t}\right]+\sigma J_{d c, i}\left[\boldsymbol{m}_{\boldsymbol{i}} \times\left[\boldsymbol{m}_{\boldsymbol{i}} \times z\right]\right],
\end{gathered}
$$

where $\gamma \approx 2 \pi \cdot 28 \mathrm{GHz} / \mathrm{T}$ is the modulus of the gyromagnetic ratio, $\mathrm{B}_{\text {eff }}$ is the effective magnetic field acting on $i$ th FL,

$$
\begin{aligned}
\boldsymbol{B}_{e f f, i}=-\mu_{0} M_{S} & \left\{\left(\begin{array}{ccc}
\kappa & 0 & 0 \\
0 & \kappa & 0 \\
0 & 0 & 1-2 \kappa
\end{array}\right) m_{i}+\right. \\
+ & \left.\left(\begin{array}{ccc}
\rho & 0 & 0 \\
0 & \rho & 0 \\
0 & 0 & -2 \rho
\end{array}\right) \boldsymbol{m}_{j}\right\},
\end{aligned}
$$

$\mu_{0}$ is the vacuum magnetic permeability, $i, j=\{t, b\}, \kappa$ and $\rho$ are respectively the selfdemagnetization and cross-demagnetization coefficients [22] dependent on the structure geometry, $\alpha$ is the Gilbert damping constant,

$$
\sigma \approx \frac{\gamma \hbar}{2 e M_{S} L}\left(\frac{3(1+P)^{3}}{4 P^{3 / 2}}-4\right)^{-1}
$$

is the current density-torque coefficient for the GMR system, $\hbar$ is the reduced Planck constant, $e$ is the modulus of the electron charge, $P$ is the dimensionless spin-polarization of the $d c$ current, while the current density $J_{d c, i}=-J_{d c}$ for the top FL and $J_{d c, i}=+J_{d c}$ for the bottom FL.

Using the spherical coordinate system for the unit vectors $\mathbf{m}_{t}=\left\{\sin \theta_{t} \cos \theta_{t}\right.$, $\left.\sin \theta_{t} \sin \theta_{t}, \cos \theta_{t}\right\}$ in the top FL and $\mathbf{m}_{b}=$ $\left\{\sin \theta_{b} \cos \theta_{b}, \sin \theta_{b} \sin \theta_{b}, \cos \theta_{b}\right\}$ in the bottom FL, respectively, we obtained from (1) the equations for the polar $\theta_{t}, \theta_{b}$ and azimuthal $\varphi_{t}, \varphi_{b}$ angles characterizing directions of the FL magnetizations. Then, we introduce new collective variables $U, V, \Phi$ and $\Psi$ :

$$
\begin{gathered}
U=\cos \theta_{t}+\cos \theta_{b}, \\
V=\cos \theta_{t}-\cos \theta_{b}, \\
\Phi=\varphi_{t}+\varphi_{b}, \\
\Psi=\varphi_{t}-\varphi_{b}
\end{gathered}
$$

and rewrite the equations describing magnetization dynamics of the system in these variables. Taking into account that the equation for the variable $U$ has a form $d U / d t=-U \cdot F(V, \Phi, \Psi)$, where $F(V, \Phi, \Psi)$ is a certain function, it is natural to assume that in a stationary regime $U \rightarrow 0$.

In the regime of stable large-angle OOP precession, the precession angles $\theta_{t}$ and $\theta_{b}$ are quite large (for instance, this was demonstrated in the numerical experiments [16, 20]) and, therefore, the variable $V^{2}=\left(\cos \theta_{i}\right.$ $\left.-\cos \theta_{b}\right)^{2}$ can be considered as a small quantity. Also, the parameters $\alpha, \kappa, \rho$ and $\sigma J_{d c} / \omega_{M}$, too, can be treated as small quantities (here $\omega_{M}=\gamma \mu_{0} M_{S}$ ). Taking this into account, we can obtain the following simplified equations describing the magnetization dynamics in the DFL STNO:

$$
\begin{gathered}
\frac{d V}{d t}=\omega_{M}\left(2 \frac{\sigma J}{\omega_{M}}-\alpha V-2 \rho \sin \Psi\right) \\
\frac{d \Psi}{d t}=-\omega_{M} V .
\end{gathered}
$$

The only singular point of the system of equations (5) is

$$
V_{0}=0, \Psi_{0}=\arcsin \left(\frac{1^{\sigma J} d c}{\rho \omega_{M}}\right) .
$$

Analyzing the behavior of the system (5) in a region near the singular point $V=V_{0}$, $\Psi=\Psi_{0}$, one can obtain the following expression for the upper dc current density threshold needed to start the generation in DFL STNO:

$$
J_{d c}^{h i g h}=\frac{\omega_{M}}{\sigma} \rho .
$$

\section{Results and discussion}

The fact that the upper threshold $J_{d c}^{h i g h}$ [see (7)] of generation is independent of damping shows that the origin of this upper threshold current is non-dissipative. In contrast the dependence of $J_{d c}^{h i g h}$ on $\rho$ means that the dipolar coupling between the FLs (its strength is characterized by the coefficient $\rho$ ) prevents the appearance of a stable magnetization precession and creates a finite $d c$ current density threshold for this precession. This, also, means that the upper current density threshold $J_{d c}^{h i g h}$ can be controlled by choosing an appropriate geometry of the system corresponding to a certain particular value of the parameter $\rho$.

For a quantitative description of the DFL STNO's operation we used the following typical parameters of the structure $[16,17$, 20 ]. We considered a symmetric circular 


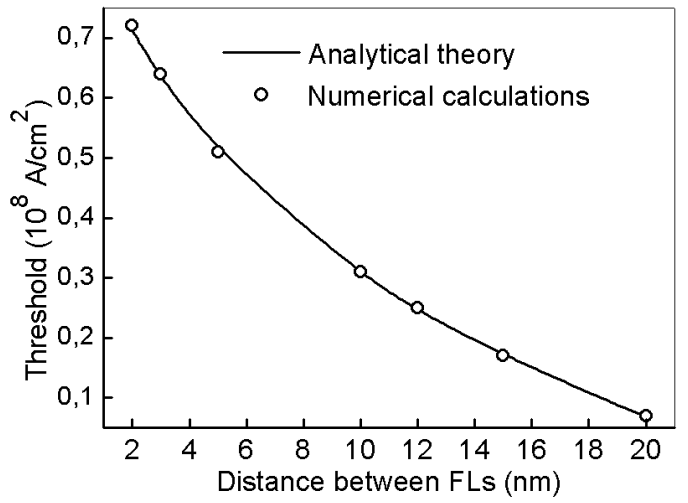

Fig. 2. Dependence of the upper $d c$ current threshold on the distance between FLs: theory (line) and numerical calculations (points).

nanopillar with radius $R=25 \mathrm{~nm}$ and $3 \mathrm{~nm}$ thick FLs $(L=3 \mathrm{~nm})$ (see Fig. 1(a)). The distance between the FLs $d$ was varied over the $2-20 \mathrm{~nm}$ range that, consequently, caused the variation of the cross-demagnetization coefficient $\rho$. We assumed the FLs have a saturation magnetization $M_{S}=$ $560 \mathrm{emu} / \mathrm{cm}^{3}\left(\mu_{0} M_{S} \approx 704 \mathrm{mT}\right)$, which is typical for thin Permalloy $\left(\mathrm{Ni}_{1-x} \mathrm{Fe}_{\chi}\right)$ films sandwiched between $\mathrm{Cu}$ layers. We assumed a value of the current polarization, $P=0.3$, typical for GMR spin valves. The coefficient of Gilbert damping $\alpha$ (the same for both FLs) was varied over the 0.002-0.09 range.

The comparison of the theoretical and numerical (from [17, 20]) results is shown in Fig. 2. As one can see, the analytical expression (7) (solid line in Fig. 2) is in good agreement with the results of numerical macrospin calculations [17, 20] (dots in Fig. 2). Taking into account that the numerical results obtained in the scope of a macrospin model [17, 20] are in good agreement with the results of micromagnetic simulations [16], when de current densities are near the upper generation threshold [17] (i.e. in the hysteresis regime of DFL STNO operation), one can safely assume that (7) is a general analytical expression for the upper $d c$ current density threshold of generation in the DFL STNO.

We believe that the obtained results are important for the development of highpower high-frequency STNOs operating in the absence of an external magnetic field.

\section{Conclusions}

An analytical theory of magnetization dynamics in a DFL STNO operating in the hysteresis regime [17] is developed. It is shown that the stationary current-induced magnetization dynamics can be characterized by two simple coupled equations for collective variables. The obtained analytical expression for the upper $d c$ current threshold of generation in DFL STNO (7) is in good agreement with the results of numerical calculations, and, evidently, demonstrates that the upper threshold current is caused by the existence of dipolar coupling between the free layers. This threshold can be controlled by choosing an appropriate geometry of the DFL STNO.

This work was supported in part by the Contract from the U.S. Army TARDEC, RDECOM, by the Grants No. ECCS1001815, ECCS-1002358, DMR-1015175 and DMR-0748810 from the National Science Foundation of the USA, by the DARPA MTO/MESO grant N66001-11-1-4114, and by the Grant No. UU34/008 from the State Fund for Fundamental Researches of Ukraine.

\section{References}

1. J.C.Slonczewski, J.Magn.Magn.Mat.D, 159, L1 (1996).

2. L.Berger, Phys. Rev. B, 54, 9353 (1996).

3. Handbook of Spin Transport and Magnetism, ed. by E.Y.Tsymbal, I.Zutic, CRC Press, New York (2012).

4. S.I.Kiselev, J.C.Sankey, I.N.Krivorotov et al., Nature (London), 425, 380 (2003).

5. A.Slavin, V.Tiberkevich, IEEE Trans. Magn., 45, 1875 (2009).

6. O.Prokopenko, E.Bankowski, T.Meitzler et al., IEEE Magn.Lett., 2, 3000104 (2011).

7. A.A.Tulapurkar, Y.Suzuki, A.Fukushima et al., Nature (London), 438, 339 (2005).

8. S.Ishibashi, T.Seki, T.Nozaki et al., Appl. Phys. Express, 3, 073001 (2010).

9. X.Cheng, C.T.Boone, J.Zhu, I.N.Krivorotov, Phys. Rev. Lett., 105, 047202 (2010).

10. O.Prokopenko, G.Melkov, E.Bankowski et al., Appl.Phys. Lett., 99, 032507 (2011).

11. O.V.Prokopenko, I.N.Krivorotov, E.Bankowski et al., J.Appl.Phys., 111, 123904 (2012).

12. O.V.Prokopenko, I.N.Krivorotov, T.J.Meitzler et al., in: Magnonics: From Fundamentals to Applications, Topics in Applied Physics, ed. by S.O.Demokritov, A.N.Slavin, Springer-Verlag, Berlin (2013), v.125.

13. J.A.Katine, E.E.Fullerton, J.Magn.Magn. Mater., 320, 1217 (2008).

14. P.M.Braganca, B.A. Gurney, B.A.Wilson et al., Nanotechnology, 21, 235202 (2010).

15. G.Rowlands, I.Krivorotov, Abstracts of the 2009 APS March Meeting, BAPS.2009 .MAR.W29.7, Pittsburgh, PA (2009). 
16. G.E.Rowlands, I.N.Krivorotov, Phys.Rev.B, 86, 094425 (2012).

17. O.V.Prokopenko, G.E.Rowlands, I.N.Krivorotov et al., in : Abstr. 12th Joint MMM-INTERMAG Conf., BR-10, Chicago, IL (2013).

18. T.Morriyama, G.Finocchio, M.Carpentieri et al., Phys. Rev. B, 86, 060411 (2012).

19. A.D.Kent, B.Ozyilmaz, E.del Barco, Appl. Phys. Lett., 84, 3897 (2004).
20. O.V.Prokopenko, I.N.Krivorotov, E.N.Bankowski et al., J.Appl.Phys., 114, 173904 (2013) [in press].

21. A.G.Gurevich, G.A.Melkov, Magnetization Oscillations and Waves, CRC Press, New York (1996).

22. O.Dmytriiev, T.Meitzler, E.Bankowski et al., J.Phys.:Condens. Matter, 22, 136001 (2010). 